\title{
Tracer Diffusion Mechanism in Amorphous Solids
}

\author{
P. K. Hung, P. H. Kien, and H. V. Hue \\ Department of Computational Physics, Hanoi University of Technology, 1 Dai Co Viet, Hanoi, Vietnam
}

Correspondence should be addressed to P. K. Hung, pkhung@fpt.vn

Received 14 September 2010; Accepted 26 October 2010

Academic Editor: Sunghak I. Lee

Copyright (๑) 2011 P. K. Hung et al. This is an open access article distributed under the Creative Commons Attribution License, which permits unrestricted use, distribution, and reproduction in any medium, provided the original work is properly cited.

\begin{abstract}
Tracer diffusion in amorphous solid is studied by mean of $n_{B}$-bubble statistic. The $n_{B}$-bubble is defined as a group of atoms around a spherical void and large bubble that represents a structural defect which could be eliminated under thermal annealing. It was found that amorphous alloys such as $\mathrm{Co}_{x} \mathrm{~B}_{100-x}(x=90,81.5$ and 70$)$ and $\mathrm{Fe}_{80} \mathrm{P}_{20}$ suffer from a large number of vacancy bubbles which function like diffusion vehicle. The concentration of vacancy bubble weakly depends on temperature, but essentially on the relaxation degree of considered sample. The diffusion coefficient estimated for proposed mechanism via vacancy bubbles is in a reasonable agreement with experiment for actual amorphous alloys. The relaxation effect for tracer diffusion in amorphous alloys is interpreted by the elimination of vacancy bubbles under thermal annealing.
\end{abstract}

\section{Introduction}

Metallic phases with amorphous structure are currently a subject of intensive studies not only due to their wide applications in practice, but also because of interest from a fundamental point of view [1-6]. Despite their technological importance, the local structure of amorphous alloys (AMAs) as well as the diffusion processes involved cannot be regarded as being fully understood. One of possible mechanisms proposed for diffusion in AMAs presents a scenario in which atoms hop into vacancy or quasivacancy determined as a microscopic void. In addition, as-quenched AMA suffers from large number of vacancies in supersaturation which quickly annihilates upon relaxation. Consequently, the diffusion constant decreases and reaches a final value until the relaxation is over, for example, the system is transposed into a well-relaxed state $[7,8]$. As such, the vacancy diffusion model enables the interpretation the relaxation effect which is observed experimentally in certain AMAs [9-13]. However, the stableness of vacancy is questioned for this model. Unlike the case of crystal, the neighbor-atom cages around vacancy are not structurally identical before and after atom hops into the vacancy. The consequent relaxation after atom jump may lead to the vacancy disappearing and finally all vacancies disappear. The molecular dynamic (MD) simulation could be used to test this question. In accordance with [14, 15], a vacancy introduced by removing some atoms from MD model disappears after one or several jumps indicating impossibility of vacancy mechanism. A logical supposition is that an elementary diffusion act will be accompanied with a large atomic arrangement around the vacancy. It results in disappearing the present vacancy, but also sometimes in the formation of a new one nearby elsewhere in the amorphous matrix. As a result, the number of vacancies in AMAs is relatively unchanged until the amorphous structure remains. Note that the number of those vacancies is not a result of thermal equilibrium of two processes like the case of crystal: new vacancies formed at the sources like free surface, dislocations, and others annihilate at the sinks. Hence, they are not thermally equilibrated vacancies of which the concentration depends on their formation energy, but "native vacancies" which always exist in amorphous structure. Overall, the vacancies in AMA are distinguished from ones in crystal. They have a different shape and size depending on the locations where vacancy resides. The detail inspection of AMA model $[5,16]$ already reveals a large number of voids with size closed to atomic radius. These voids could be "native vacancies". However, the result in these works depends on the atomic radius which is not uniquely determined yet. 
TABLE 1: The parameters of the interatomic potential (1).

\begin{tabular}{lcccccc}
\hline Pairs & $a\left(\mathrm{eV} / \AA^{4}\right)$ & $b(\AA)$ & $c\left(\mathrm{eV} / \AA^{2}\right)$ & $d(\AA)$ & $e(\mathrm{eV})$ & $r_{\text {cutoff }}(\AA)$ \\
\hline Co-Co & -0.12812 & -1.82709 & 1.15421 & -2.50849 & -0.13448 & 3.44 \\
Co-B & -0.10967 & -1.47709 & 0.98799 & -2.15849 & -0.11511 & 3.09 \\
B-B & -0.08772 & -2.17709 & 0.79028 & -2.85849 & -0.09208 & 3.79 \\
Fe-Fe & -0.18892 & -1.82709 & 1.70192 & -0.50849 & -0.19829 & 3.44 \\
Fe-P & -0.31411 & -058709 & 2.82978 & -2.26849 & -0.32970 & 3.20 \\
P-P & -0.07543 & -2.60709 & 0.64791 & -3.27885 & -0.07531 & 4.21 \\
\hline
\end{tabular}

In other diffusion models [17-21], the thermally activated process for diffusion in AMA can be assisted by certain kinds of structural defects, but may involve more atoms besides the diffusing atom. Accordingly, several atoms around a defect move by a small distance such that diffusing atom can move over a large distance. This approach in fact employs a combination of liquid and defect-mediated diffusion models [18]. The common point of those approaches is that AMA suffers from a number of "favored sites" and restriction for atoms to move out of their neighbor-atom cages. The transport therefore is by thermal activated hoping of neighbor atom into those sites. However, up to now, no work has been done in order to clarify the "favored sites" in the amorphous structure which is easily prepared using available simulation techniques.

In this work an attempt is made to address the question in term of "favored sites" or quasivacancy where diffusing atom can jump into overcoming a corresponding potential barrier. The tetrahedrons constructing the structure of AMA are used to clarify these quasi-vacancies based on the analysis of profile of potential energy for neighbor atoms. To clarify this problem several large models of $\mathrm{Co}_{x} \mathrm{~B}_{100-x}$ and $\mathrm{Fe}_{80} \mathrm{P}_{20}$ consisting of $2 \cdot 10^{5}$ atoms have been constructed. The relaxation after atom hop into the quasi-vacancy is conducted and analyzed accordingly.

\section{Calculation Procedure}

Amorphous model can be prepared by mean of $\mathrm{MD}$ simulation, but this method consumes much computing time, especially for constructing and relaxing a large model. Hence, in the present work we use a continuous static relaxation (SR) technique, the cheaper computing method which provides a structure of AMA almost similar to MD model at room temperature. According to SR method each atom moves in the direction of force acting on it from all remaining atoms by a defined length $d r$. This movement of all atoms is repeated many times until the system reaches an equilibrated state, for example, the total potential energy of system fluctuates around a constant value and the pressure is almost equal to zero. More details about SR method can be found elsewhere [22]. The initial configuration is generated by random distribution of all atoms in a cubic cube with periodic boundary conditions. The density is adopted from real amorphous alloys. We employ the Paka-Doyama type potentials given as follows:

$$
U(r)= \begin{cases}a(r+b)^{4}+c(r+d)^{2}+e, & 0 \leq r \leq r_{\text {cutoff }}, \\ 0, & r_{\text {cutoff }}<r,\end{cases}
$$

where $r$ is the inter-atomic distance and in $\AA ; U(r)$ is in $\mathrm{eV}$. The parameters of potentials (1) are given in Table 1 .

Eight SR models with composition $\mathrm{Co}_{x} \mathrm{~B}_{100-x}$ and $\mathrm{Fe}_{80} \mathrm{P}_{20}$ have been constructed. Among them the models $A_{1}, A_{2}$ and $A_{3}$ present three possible metastable states of $\mathrm{Co}_{81.5} \mathrm{~B}_{18.5}$ with the same density. More stable configurations (model $\mathrm{A}_{2}$ and $\mathrm{A}_{3}$ ) are obtained by "shaking procedure": the model $\mathrm{A}_{1}$ is relaxed with $d r=0.4 \AA$ over several hundred SR steps and then obtained sample is treated with $d r=0.01 \AA$ until the system attains a new equilibrium. The shaking procedure is repeated many times to obtain corresponding models $\mathrm{A}_{2}$, and $\mathrm{A}_{3}$.

It is well known that as-quenched sample of AMA undergoes a structural relaxation under thermal annealing and transposes into well-relaxed sample with higher density. To account this effect, we prepare two models $B_{1}$ and $B_{2}$ with the density higher by 0.3 and 0.6 percent than the density of model $A_{1}$. The preparing model $B_{1}$ and $B_{2}$ are performed by similar way as done for model $A_{1}$. The models $B_{i}$ as well as model $A_{i}$ represents the different metastable state of amorphous alloy $\mathrm{Co}_{81.5} \mathrm{~B}_{18.5}$.

Amorphous structure as mentioned above suffers from a number of different kinds of voids being a "favored site" or vacancy/interstice. Consider some types of atomic cage which may be a "favored site". The microscopic atom cage demonstrated in Figure 1 is determined in the following way: four neighboring atoms form a tetrahedron and have a circum-sphere (CS) of which surface passes through the vertices of this tetrahedron. Consider only such tetrahedron that its CS does not contain any atom inside, for example, those atoms are the nearest neighboring. Let $R_{B}$ and $n_{B}$ be the radius of CS and the number of atoms on the surface of CS, respectively. The atom on surface of CS is determined as one that is located from the center of CS at a distance in range of $R_{B} \pm 0.1 \AA$. The number $n_{B}$ varies from 4 to 9 depending on the concrete location in amorphous structure. The microscopic atom cage just described likes a bubble (a group of atoms around a spherical void) and hereafter we call it $n_{B}$-bubble and, radius $R_{B}$ 




(a)

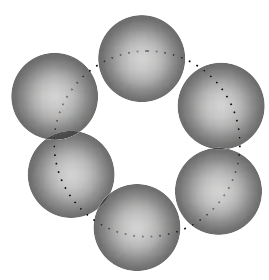

(b)

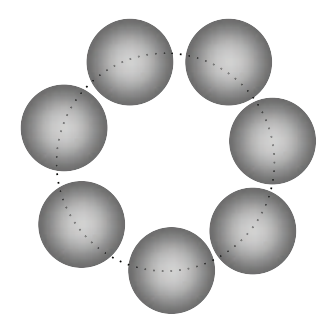

(c)



(d)



(e)

Figure 1: The schematic illustration of 4-bubble (a), 6-bubble (b), 7-bubble (c), the region with 5-bubble before (d), and after completing the atom jump and relaxation which causes the creation of new bubble nearby (e); the dot-sided circles represent the CS.

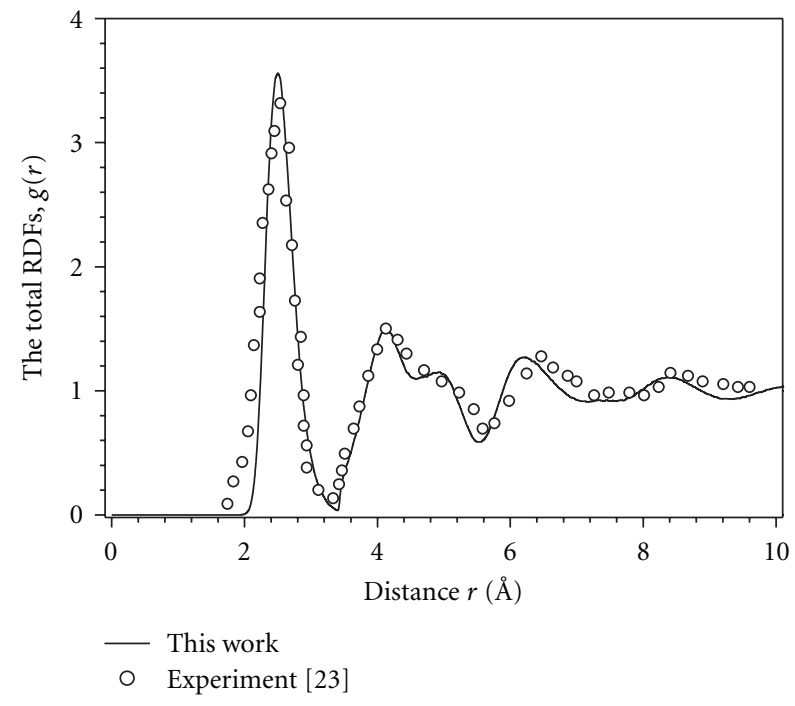

(a)



(b)

FIgURE 2: The total radial distribution function $g(r)$ (left) and structure factor (right) of amorphous alloys $\mathrm{Co}_{81.5} \mathrm{~B}_{18.5}$.

is denoted to bubble radius. Several kinds of $n_{B}$-bubble schematically illustrated in Figure 1 are shown in Figure 5. If one among $n_{B}$ atoms can move into center of CS, then this $n_{B}$-bubble play a role of vacancy or the "favored site". Only the bubble with $n_{B}$ bigger than 4 is taken into account.
To evaluate the concentration of $n_{B}$-bubble, the next calculation has performed as follows: firstly, we detect all $n_{B}$ bubbles in constructed models. Secondly, we find the vacancy bubble, for example, the $n_{B}$-bubble in which the neighboring atom can jump overcoming the potential energy barrier. To detect $n_{B}$-bubbles we take all sets of four atoms in the system. 


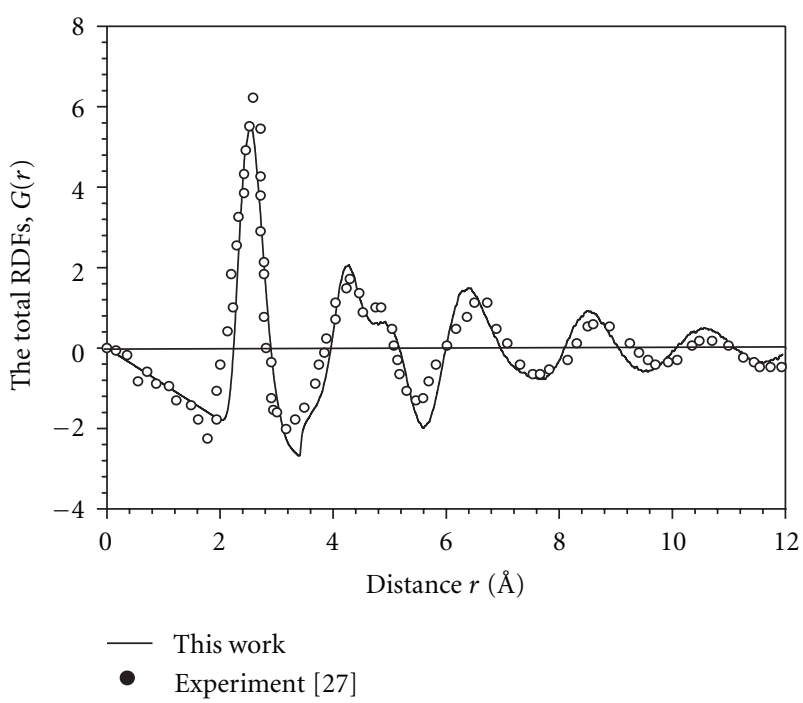

(a)



(b)

FIGURE 3: The total reduced distribution function $G(r)=4 \pi r \rho_{o}\left(g(r)-1\right.$ (b) and structure factor (a) of amorphous alloys Fe ${ }_{80} \mathrm{P}_{20}$.

TABLE 2: The characteristics of constructed models; $\rho$ is the density and $\varepsilon$ the energy per atom.

\begin{tabular}{lcccccccc}
\hline Model & $\mathrm{A}_{1}$ & $\mathrm{~A}_{2}$ & $\mathrm{~A}_{3}$ & $\mathrm{~B}_{1}$ & $\mathrm{~B}_{2}$ & $\mathrm{C}$ & $\mathrm{D}$ & $\mathrm{E}$ \\
\hline$\rho\left(\mathrm{g} / \mathrm{cm}^{3}\right)$ & 8.309 & 8.309 & 8.309 & 8.328 & 8.349 & 8.529 & 7.979 & 7.228 \\
$\varepsilon(\mathrm{eV})$ & -0.9187 & -0.9301 & -0.9381 & -0.9343 & -0.9334 & -0.9238 & -0.9535 & -1.7025 \\
\hline
\end{tabular}

The distance between any pair atoms of those sets is less than a specified value. Then the CS is built and the number $n_{B}$ is calculated.

\section{Result and Discussion}

We first report on the validity of our models by comparing the simulation result and experimental scattering data. The comparison has been carried out by both total pair radial distribution function $g(r)$ and structural factor $a(k)$ as shown in Figures 2 and 3. In general, a satisfactory agreement with experiment is observed here. The main characteristics of those models are listed in Tables 2 and 3. Here $A_{1}, A_{2}, A_{3}, B_{1}$, and $B_{2}$ are models $C_{81.5} B_{18.5}$; $\mathrm{C}, \mathrm{D}$, and $\mathrm{E}$ correspond to the model $\mathrm{Co}_{90} \mathrm{~B}_{10}, \mathrm{Co}_{70} \mathrm{~B}_{30}$, and $\mathrm{Fe}_{80} \mathrm{P}_{20}$, respectively. One can see that the calculated position, and height of $g_{i j}(r)$ as well as pair averaged coordination number $z_{i j}$ are very close to experimental data demonstrating that although the Paka-Doyama type potentials are simple, they reproduce well the structure of metal-metalloid AMA. Therefore, our models can be used for further study of AMAs. The pair radial distribution functions (PRDFs) $g_{i j}(r)$ plotted in Figure 4 show a split of second peak that is believed to be the structural feature of AMA. For models $A_{1}, A_{2}$, and $A_{3}$ presenting the different metastable states of AMA $\mathrm{Co}_{81.5} \mathrm{~B}_{18.5}$, their PRDFs is almost the same although the difference of potential energy per atom of those models is about 4 percents. The similar trend is observed for models $A_{1}, B_{1}$, and $B_{2}$ which differ significantly in the density.
Figure 5 shows some typical $n_{B}$-bubbles and the number of different kinds of them is listed in Table 4. There is a tendency to decrease in the number of bubbles from model $\mathrm{A}_{1}$ (less stable configuration) to model $\mathrm{A}_{3}$ (most stable configuration). The comparison between models with different density (model $A_{1}, B_{1}$, and $B_{2}$ ) also shows that the lower model density, the larger number of bubbles. Because as-quenched AMA has lower density and it is a less stable configuration in comparison with well-relaxed sample, hence our calculation indicates that the number of bubbles in asquenched sample is bigger than one of well-relaxed sample. It means that the bubbles represent the structural defect of quenched nature, and their number decreases during the thermal annealing. Regarding the metalloid contain for system $\mathrm{Co}_{x} \mathrm{~B}_{100-x}$, we observe a monotonous increase in the number of 5-, 6-, and 7-bubbles with increasing the boron contain. The model $\mathrm{Fe}_{80} \mathrm{P}_{20}$ contains larger number of bubbles compared to system $\mathrm{Co}_{x} \mathrm{~B}_{100-x}$.

Important quantity which characterized the $n_{B}$-bubbles is their radius distribution shown in Table 5. Here one can see a number of bubbles with radius bigger than $2.0 \AA$. Obviously these bubbles could play a role like vacancy, for example, its volume is enough large to allow a neighbor atom to jump into it.

Now we turn to clarify the importance of $n_{B}$-bubbles for tracer diffusion. For every $n_{B}$-bubble we move one among $n_{B}$ neighboring atoms step by step into its CS's center (see Figures $1(\mathrm{~d}), 1(\mathrm{e}))$. The step length is equal to $0.02 \AA$. Then the potential energy profile (EPP) is determined and recorded. Several typical EPPs are presented in Figure 6. 
TABLE 3: Structural characteristics of amorphous $\mathrm{Co}_{100-x} \mathrm{~B}_{x}$ and $\mathrm{Fe}_{80} \mathrm{P}_{20}$ alloys; where $g_{i j}, r_{i j}$ is the height and position of first peak of $g_{i j}(r)$; $z_{i j}$ is the averaged coordination number; $1-1,1-2,2-1$ and 2-2 are the M-M, M-Me, Me-M and Me-Me pairs, respectively.

\begin{tabular}{|c|c|c|c|c|c|c|c|c|c|c|}
\hline \multirow{2}{*}{ Model } & \multicolumn{3}{|c|}{$r_{i j}(\AA)$} & \multicolumn{3}{|c|}{$g_{i j}$} & \multicolumn{4}{|c|}{$z_{i j}$} \\
\hline & $1-1$ & $1-2$ & $2-2$ & $1-1$ & $1-2$ & $2-2$ & $1-1$ & $1-2$ & $2-1$ & $2-2$ \\
\hline$\overline{A_{1}}$ & 2.50 & 2.08 & 3.06 & 3.44 & 3.77 & 3.96 & 12.21 & 2.12 & 9.34 & 4.43 \\
\hline $\mathrm{A}_{2}$ & 2.50 & 2.08 & 3.02 & 3.50 & 3.86 & 3.88 & 12.22 & 2.13 & 9.38 & 4.48 \\
\hline $\mathrm{A}_{3}$ & 2.50 & 2.08 & 3.08 & 3.57 & 3.99 & 4.04 & 12.22 & 2.13 & 9.39 & 4.52 \\
\hline $\mathrm{B}_{1}$ & 2.50 & 2.08 & 3.04 & 3.54 & 3.99 & 4.03 & 12.24 & 2.13 & 9.39 & 4.51 \\
\hline $\mathrm{B}_{2}$ & 2.48 & 2.06 & 3.06 & 3.52 & 3.93 & 4.18 & 12.27 & 2.14 & 9.42 & 4.55 \\
\hline $\mathrm{Co}_{81.5} \mathrm{~B}_{18.5}$ & $2.50^{\mathrm{a}}$ & $2.10^{\mathrm{b}}$ & $3.25^{c}$ & - & - & - & $12.70^{\mathrm{a}}$ & $1.50^{\mathrm{a}}$ & $6.606^{\mathrm{a}}$ & - \\
\hline $\mathrm{C}$ & 2.50 & 2.10 & 3.06 & 3.58 & 3.95 & 4.43 & 12.85 & 1.07 & 9.62 & 2.41 \\
\hline $\mathrm{D}$ & 2.54 & 2.10 & 3.04 & 3.37 & 3.86 & 3.43 & 11.30 & 3.82 & 8.92 & 7.51 \\
\hline E & 2.58 & 2.36 & 3.62 & 3.64 & 5.91 & 2.36 & 10.56 & 2.55 & 10.2 & 3.45 \\
\hline $\mathrm{Fe}_{80} \mathrm{P}_{20} \mathrm{~g}$ & 2.61 & 2.38 & 3.50 & - & - & - & 10.40 & 2.60 & 8.10 & 3.50 \\
\hline $\mathrm{Fe}_{80} \mathrm{P}_{20}{ }^{\mathrm{h}}$ & 2.60 & 2.35 & 3.50 & 3.58 & 5.80 & 2.41 & 10.41 & 2.51 & 10.4 & 4.89 \\
\hline
\end{tabular}

Experimental data $-{ }^{\mathrm{a}}[23] .{ }^{\mathrm{b}}[24] .{ }^{\mathrm{c}}[24,25] .{ }^{\mathrm{g}}[26,27] .{ }^{\mathrm{h}}[28]$.

TABLE 4: The number of $n_{B}$-bubbles per atom.

\begin{tabular}{|c|c|c|c|c|c|c|c|}
\hline \multirow{2}{*}{ Model } & \multicolumn{5}{|c|}{ Number of $n_{B}$-bubbles per atom } & \multicolumn{2}{|c|}{ The number of VBs } \\
\hline & $5(\times 1)$ & $6\left(\times 10^{-1}\right)$ & $7\left(\times 10^{-3}\right)$ & $8\left(\times 10^{-3}\right)$ & $9\left(\times 10^{-3}\right)$ & $\mathrm{m}_{\mathrm{M}}\left(\times 10^{-4}\right)$ & $\mathrm{m}_{\mathrm{Me}}\left(\times 10^{-4}\right)$ \\
\hline$\overline{\mathrm{A}_{1}}$ & 1.1082 & 1.5441 & 5.4551 & 0.7952 & 0.1451 & 12.4503 & 26.9002 \\
\hline $\mathrm{A}_{2}$ & 1.0737 & 1.5116 & 4.9350 & 0.6251 & 0.0103 & 4.7513 & 17.7013 \\
\hline $\mathrm{A}_{3}$ & 1.0572 & 1.3598 & 2.0651 & 0.2552 & 0.0150 & 0.8504 & 10.4502 \\
\hline $\mathrm{B}_{1}$ & 1.0537 & 1.4056 & 4.5653 & 0.1404 & 0.0008 & 1.6521 & 11.7532 \\
\hline $\mathrm{B}_{2}$ & 1.0380 & 1.3223 & 2.8450 & 0.2300 & 0 & 0.9501 & 9.0503 \\
\hline $\mathrm{C}$ & 0.7411 & 1.6437 & 2.1302 & 1.5703 & 0 & 2.9024 & 9.4002 \\
\hline $\mathrm{D}$ & 1.7349 & 1.6563 & 9.7801 & 0.7702 & 0 & 7.8043 & 13.8045 \\
\hline $\mathrm{E}$ & 1.0378 & 2.4913 & 17.6403 & 1.8504 & 0 & 4.6041 & 1.0031 \\
\hline
\end{tabular}

TABLe 5: The radii distribution of bubbles.

\begin{tabular}{|c|c|c|c|c|c|c|c|c|}
\hline$R_{B}, \AA$ & $A_{1}$ & $\mathrm{~A}_{2}$ & $\mathrm{~A}_{3}$ & $\mathrm{~B}_{1}$ & $\mathrm{~B}_{2}$ & $\mathrm{C}$ & $\mathrm{D}$ & $E$ \\
\hline 1.4 & 0.0416 & 0.0415 & 0.0425 & 0.0460 & 0.0507 & 0.0122 & 0.1176 & 0 \\
\hline 1.5 & 0.3369 & 0.3518 & 0.3626 & 0.3629 & 0.3711 & 0.1236 & 0.8188 & 0.0005 \\
\hline 1.6 & 0.3780 & 0.3781 & 0.3575 & 0.3628 & 0.3621 & 0.2167 & 0.6044 & 0.0829 \\
\hline 1.7 & 0.2739 & 0.2504 & 0.2394 & 0.2379 & 0.2259 & 0.2985 & 0.2457 & 0.3391 \\
\hline 1.8 & 0.1979 & 0.1834 & 0.1784 & 0.1716 & 0.1504 & 0.2335 & 0.1059 & 0.4797 \\
\hline 1.9 & 0.0281 & 0.0186 & 0.0115 & 0.0139 & 0.0100 & 0.0178 & 0.0150 & 0.1898 \\
\hline 2.0 & 0.0101 & 0.0060 & 0.0031 & 0.0036 & 0.0027 & 0.0057 & 0.0036 & 0.1104 \\
\hline 2.1 & 0.0018 & 0.0012 & 0.0004 & 0.0002 & 0.0003 & 0.0013 & 0.0001 & 0.0460 \\
\hline 2.2 & 0.0007 & 0.0002 & 0 & 0.0001 & 0 & 0.0001 & 0.0001 & 0.0219 \\
\hline 2.3 & 0.0002 & 0 & 0 & 0.0001 & 0 & 0.0001 & 0 & 0.0136 \\
\hline 2.4 & 0.0001 & 0 & 0 & 0 & 0 & 0 & 0 & 0.0081 \\
\hline 2.5 & 0 & 0 & 0 & 0 & 0 & 0 & 0 & 0.0051 \\
\hline 2.6 & 0 & 0 & 0 & 0 & 0 & 0 & 0 & 0.0041 \\
\hline 2.7 & 0 & 0 & 0 & 0 & 0 & 0 & 0 & 0.0028 \\
\hline 2.8 & 0 & 0 & 0 & 0 & 0 & 0 & 0 & 0.0014 \\
\hline 2.9 & 0 & 0 & 0 & 0 & 0 & 0 & 0 & 0.0009 \\
\hline 3.0 & 0 & 0 & 0 & 0 & 0 & 0 & 0 & 0.0004 \\
\hline
\end{tabular}






(a)



(c)

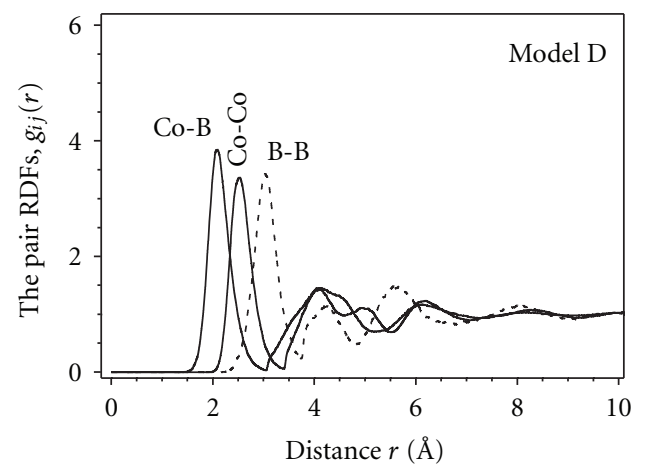

(b)

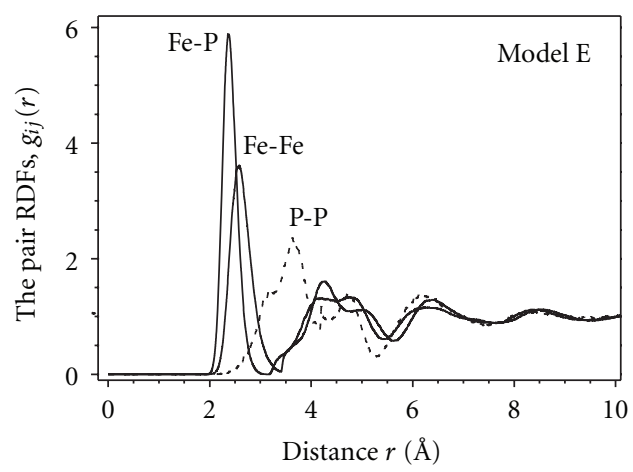

(d)

FIgURE 4: The pair radial distribution function.
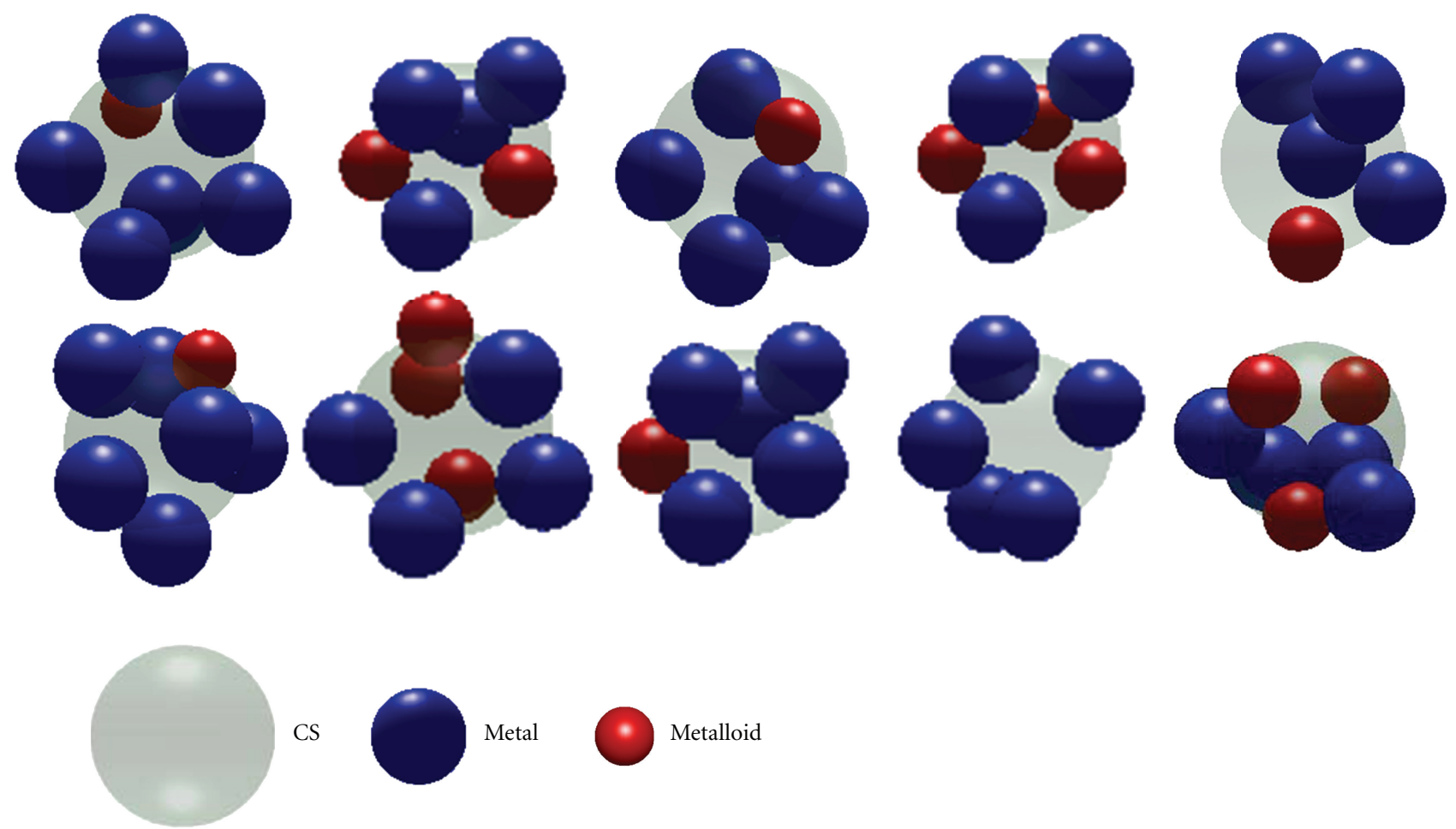

CS

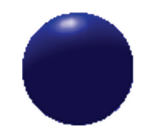

Metal

Metalloid

FIGURE 5: The typical $n_{B}$-bubbles detected in the constructed models which consist of a CS and several neighboring atoms on their surface. 
TABLE 6: The averaged square displacement upon atomic jump and relaxation. Here $\left\langle x^{2}{ }_{M}\right\rangle$ and $\left\langle x^{2}{ }_{\text {Me }}\right\rangle$ are in $\AA^{2}$.

\begin{tabular}{llcccccccc}
\hline \multicolumn{1}{c}{ Model } & & $\mathrm{A}_{1}$ & $\mathrm{~A}_{2}$ & $\mathrm{~A}_{3}$ & $\mathrm{~B}_{1}$ & $\mathrm{~B}_{2}$ & $\mathrm{C}$ & $\mathrm{D}$ & $\mathrm{E}$ \\
\hline Metal atomic & $\left\langle x^{2}{ }_{\mathrm{M}}\right\rangle$ & 8.951 & 6.107 & 6.202 & 7.045 & 6.075 & 6.463 & 6.936 & 6.629 \\
jump & $\left\langle x^{2}{ }_{\mathrm{Me}}\right\rangle$ & 1.164 & 0.193 & 0.184 & 0.853 & 0.175 & 0.572 & 0.219 & 0.077 \\
Metalloid & $\left\langle x^{2}{ }_{\mathrm{M}}\right\rangle$ & 1.834 & 0.807 & 0.576 & 0.638 & 0.589 & 0.794 & 0.791 & 0.630 \\
atomic jump & $\left\langle x^{2}{ }_{\mathrm{Me}}\right\rangle$ & 4.947 & 4.332 & 4.301 & 4.125 & 4.109 & 4.201 & 4.301 & 5.235 \\
\hline
\end{tabular}

TABLE 7: The diffusion coefficients in amorphous alloys at 570-640 K obtained by the simulation and experiment; M-Co or Fe and Me-B or P.

\begin{tabular}{|c|c|c|c|c|c|}
\hline System & $\mathrm{M}$ & $\mathrm{D}_{\mathrm{M}}, \mathrm{m}^{2} \mathrm{~s}^{-1}$ & $\mathrm{Me}$ & $\mathrm{D}_{\mathrm{Me}}, \mathrm{m}^{2} \mathrm{~s}^{-1}$ & Reference \\
\hline Model $A_{1}$ & Co & $1.4 \times 10^{-20}-1.0 \times 10^{-19}$ & $\mathrm{~B}$ & $1.8 \times 10^{-19}-1.2 \times 10^{-18}$ & This work \\
\hline Model $\mathrm{A}_{2}$ & Co & $1.4 \times 10^{-21}-1.3 \times 10^{-20}$ & B & $1.4 \times 10^{-20}-1.3 \times 10^{-19}$ & This work \\
\hline Model $\mathrm{A}_{3}$ & Co & $2.1 \times 10^{-22}-1.9 \times 10^{-21}$ & B & $1.9 \times 10^{-20}-1.6 \times 10^{-19}$ & This work \\
\hline Model $B_{1}$ & Co & $1.4 \times 10^{-22}-1.4 \times 10^{-21}$ & B & $8.5 \times 10^{-21}-8.1 \times 10^{-20}$ & This work \\
\hline Model $\mathrm{B}_{2}$ & Co & $1.9 \times 10^{-22}-1.6 \times 10^{-21}$ & B & $1.4 \times 10^{-20}-1.1 \times 10^{-19}$ & This work \\
\hline Model C & Co & $4.5 \times 10^{-21}-3.1 \times 10^{-20}$ & B & $5.7 \times 10^{-20}-4.0 \times 10^{-19}$ & This work \\
\hline Model D & Co & $6.3 \times 10^{-23}-6.9 \times 10^{-22}$ & B & $8.2 \times 10^{-22}-8.8 \times 10^{-21}$ & This work \\
\hline Model E & $\mathrm{Fe}$ & $2.6 \times 10^{-22}-1.2 \times 10^{-21}$ & $\mathrm{P}$ & $2.7 \times 10^{-23}-4.7 \times 10^{-21}$ & This work \\
\hline $\mathrm{Gb}_{16} \mathrm{Co}_{84}$ & Co & $2.5 \times 10^{-22}-3.6 \times 10^{-21}$ & - & - & {$[3]$} \\
\hline $\mathrm{Co}_{79} \mathrm{Nb}_{14} \mathrm{~B}_{7}$ & Co & $1.8 \times 10^{-22}-1.1 \times 10^{-20}$ & - & - & {$[29]$} \\
\hline $\mathrm{Co}_{89} \mathrm{Zr}_{11}$ & Co & $2.4 \times 10^{-21}-7.3 \times 10^{-20}$ & - & - & {$[30]$} \\
\hline $\mathrm{Fe}_{80} \mathrm{~B}_{20}$ & $\mathrm{Fe}$ & $2.2 \times 10^{-23}-5.2 \times 10^{-21}$ & - & - & {$[2]$} \\
\hline $\mathrm{Fe}_{40} \mathrm{Ni}_{40} \mathrm{~B}_{20}$ & $\mathrm{Fe}$ & $4.7 \times 10^{-23}-8.8 \times 10^{-21}$ & B & $1.7 \times 10^{-21}-1.4 \times 10^{-19}$ & {$[31,32]$} \\
\hline $\mathrm{Fe}_{40} \mathrm{Ni}_{40} \mathrm{~B}_{20}$ & - & - & $\mathrm{P}$ & $1.3 \times 10^{-23}-8.4 \times 10^{-21}$ & {$[33]$} \\
\hline $\mathrm{Fe}_{40} \mathrm{Ni}_{40} \mathrm{P}_{14} \mathrm{~N}_{6}$ & - & - & $\mathrm{P}$ & $4.2 \times 10^{-23}-3.2 \times 10^{-21}$ & {$[34]$} \\
\hline
\end{tabular}



FIGURE 6: Potential energy profile of atoms moving from their site to the center of the CS of VB in $\mathrm{Co}_{81.5} \mathrm{~B}_{18.5}$, and $\mathrm{Fe}_{80} \mathrm{P}_{20}$.

It is clear that there are two kinds of EPPs: first one corresponds to EPPs presented by curves $a, b, c, d$, and $e$ where the energy of diffusing atom (DA) increases until it attains a maximum and then decreases. They show a characteristic EPP for atom jumping into vacancy over a potential barrier. Hereafter those bubbles are denoted to vacancy bubbles (VBs). The second kind of EPP presented by curve $f$ shows a monotonous increase of potential energy of DA. In this case, the atom cannot jump into a bubble because of very high potential barrier. The site energy and barrier distributions of diffusing atom of $\mathrm{VB}$ are shown in Figure 7. It can be seen that they are spread in very wide range. Therefore, the VBs have not only different shape and number $n_{B}$, but also different potential barriers depending on the location where they reside. The number of VBs found in considered models is listed in Table 2. There are two kinds of VBs: the metal and metalloid VB where metal or metalloid atom can jump into VB, respectively. Figure 8 presents the VB's distribution in simulation box. It is interesting to note that some VBs are grouped into a large cluster which may consist of about $50 \mathrm{VBs}$ (see model $\mathrm{E}$ in Figure 8).

In the case of crystal lattice as atom jumps it leaves behind a new vacancy and only the atom just jumped had an essential displacement. For AMA the jump of DA does not leave a new VB, but due to the relaxation resulting in the strong atomic arrangement a new VB is created nearby. As a result, the number of VBs is relatively unchanged and many atoms are involved in the collective movement. To account for this effect, we replace the neighboring atom into the VB and then relax the whole system until a new equilibrium has been established. The square displacement of atoms after the jump, $x_{\mathrm{M}}{ }^{2}$ and $x_{\mathrm{Me}^{2}}$, are shown in Figure 9 and the average their value is listed in Table 6. 




(a)

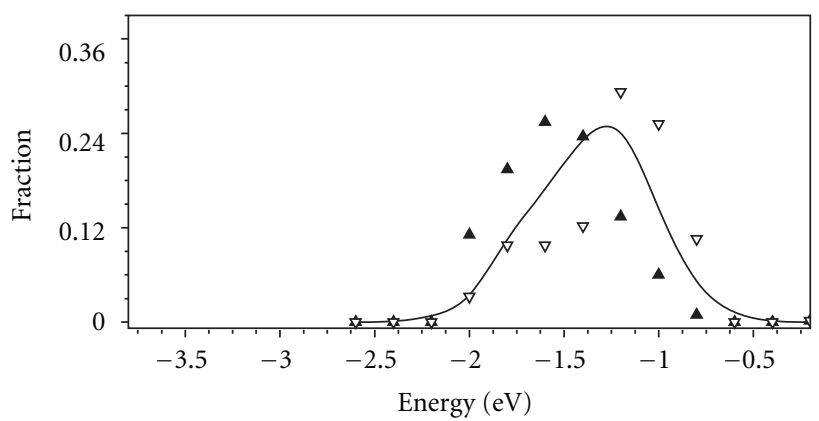

$\begin{array}{ll}- & \mathrm{A}_{1} \\ \nabla & \mathrm{C} \\ \boldsymbol{\Delta} & \mathrm{D}\end{array}$

(c)

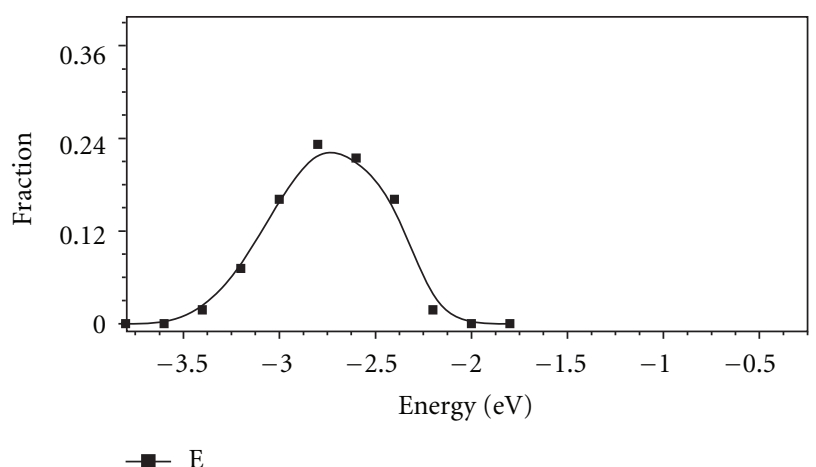

(e)


(b)



$-\mathrm{A}_{1}$

$\nabla \quad \mathrm{C}$

$\Delta \mathrm{D}$

(d)



(f)

FIgURE 7: The distributions of site energy (left) and potential energy barrier (right) for DAs in $\mathrm{Co}_{100-x} \mathrm{~B}_{x}$ and $\mathrm{Fe}_{80} \mathrm{P}_{20}$.

Here $x_{\mathrm{M}}^{2}$ and $x_{\mathrm{Me}}{ }^{2}$ are the square displacement of metal and metalloid atoms. It can be seen that the parameter $x_{\mathrm{M}}^{2}$ and $x_{\mathrm{Me}}^{2}$ varies in very wide range from 0.5 to $15 \AA^{2}$. Because the jump length of DA is about 1-2 $\AA$, so a large value of both $x_{\mathrm{M}}{ }^{2}$ and $x_{\mathrm{Me}}{ }^{2}$, for example, $x_{\mathrm{M}}{ }^{2}=11 \AA^{2}$ and $x_{\mathrm{Me}}^{2}=9 \AA^{2}$ evidences the collective movement of large number of atoms. Furthermore, the model $A_{1}$ (less stable configuration) undergoes a stronger arrangement in comparison with the model $\mathrm{A}_{3}$ (most stable configuration).
Such one can distinguish two diffusion mechanisms: first one like collective mechanism where atoms jump into VB and relaxation makes a strong arrangement spread over large region. The concentration of VBs spontaneously decreases during the thermal annealing. This mechanism occurs in the less stable model with low density (as-quenched sample). The second mechanism occurs in more stable model with higher density (well-relaxed sample) presenting likely usual vacancy mechanism. 


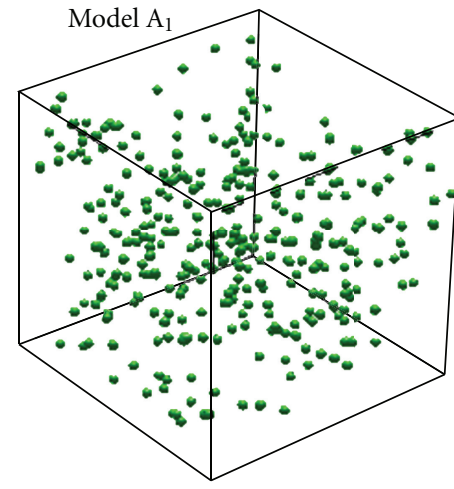

(a)

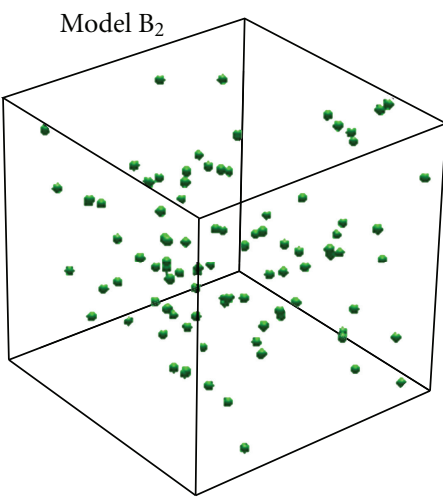

(d)



(b)

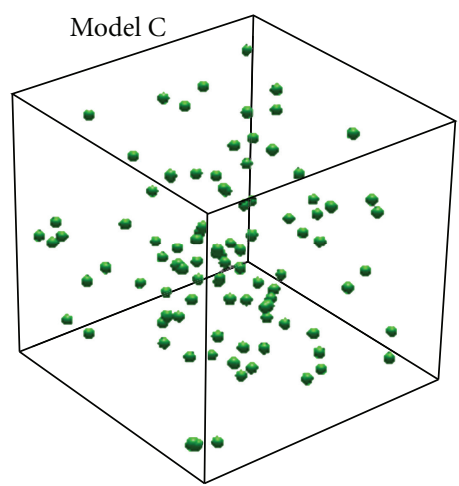

(e)

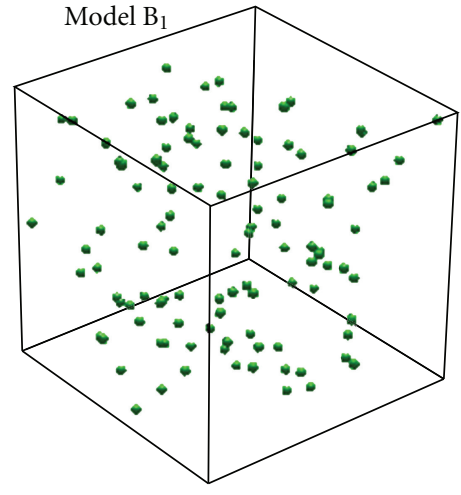

(c)

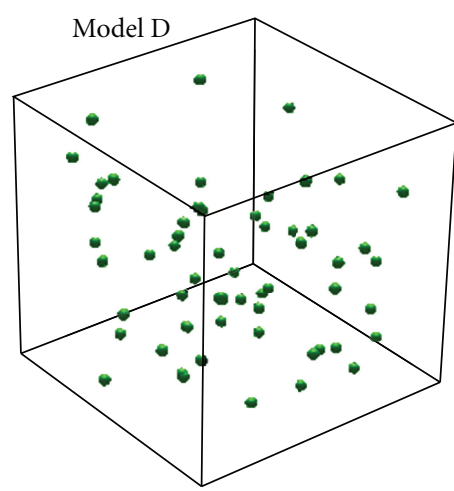

(f)

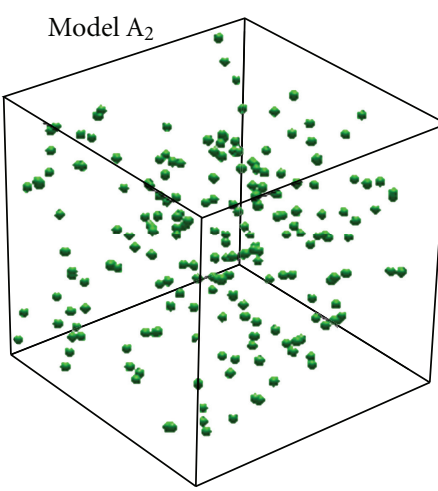

(g)



(h)

FIGURE 8: The VB's distribution in simulation box of constructed models; the sphere represents the CS of VB.

The number of VBs has been calculated after atom jump and completing the relaxation. The result obtained here shows that some bubbles transpose into VB and sometime the converse trend is observed where the VB transpose into small bubbles. The total number of $\mathrm{VB}$ changes in the range of \pm 15 depending on the location where the DA jump occurs. The diffusion coefficient for diffusion via VB can be estimated as

$$
D_{S}=\frac{1}{6} f \frac{1}{N_{\text {Atom }}}\left[\sum_{i}^{m_{\mathrm{M}}} \varphi_{\mathrm{Mi}} x_{S i}^{2}+\sum_{i}^{m_{\mathrm{Me}}} \varphi_{\mathrm{Mei}} x_{S i}^{2}\right],
$$

where $S$ is denoted to $\mathrm{Co}, \mathrm{Fe}, \mathrm{B}$, or $\mathrm{P}$ atoms; $m_{\mathrm{M}}$ and $m_{\mathrm{Me}}$ are number of metal and metalloid VBs; $N_{\text {Atom }}$ is the total number of atoms; $f$ is the correlation factor for consecutive hops. The effective completion frequency $\varphi_{S i}$ is given as

$$
\varphi_{S i}=\nu_{0} \exp \left(\frac{s_{S i}}{k_{B}}\right) \exp \left(-\frac{h_{S i}}{k_{B} T}\right) .
$$

Here $s_{S i}, h_{S i}$ denotes the entropy change and migration enthalpy, $k_{B}$ is the Boltzmann constant, $T$ is the temperature, and $v_{o}$ is the attempt frequency.

Assuming $\exp \left(s_{m} / k_{B}\right) \approx 1, f=1$ and taking the $m_{\mathrm{M}}$ and $m_{\mathrm{Me}}$ from Table 2 the diffusion constant is determined in the temperature range of 570-640 K and listed in Table 7. As can be seen, the result is well consistent with experiment for actual alloys. 


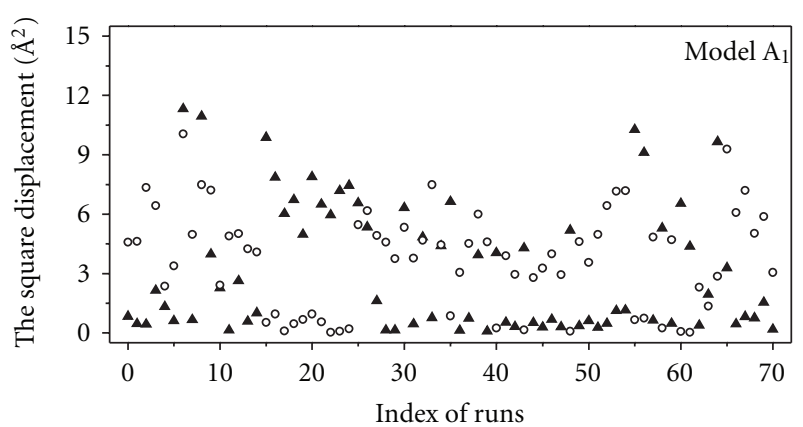

(a)

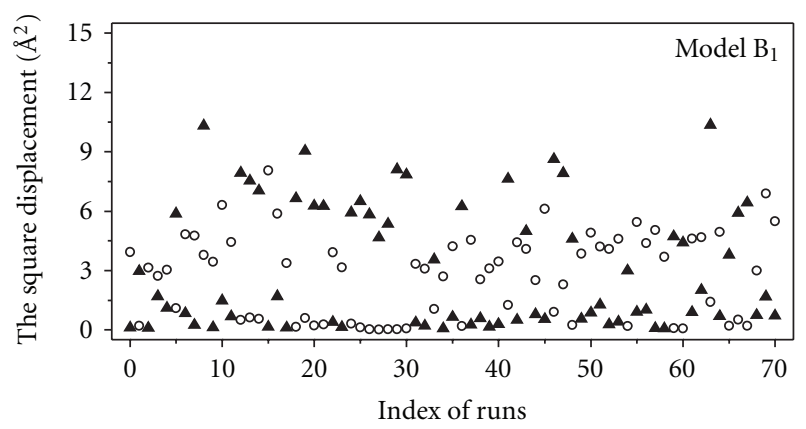

(c)

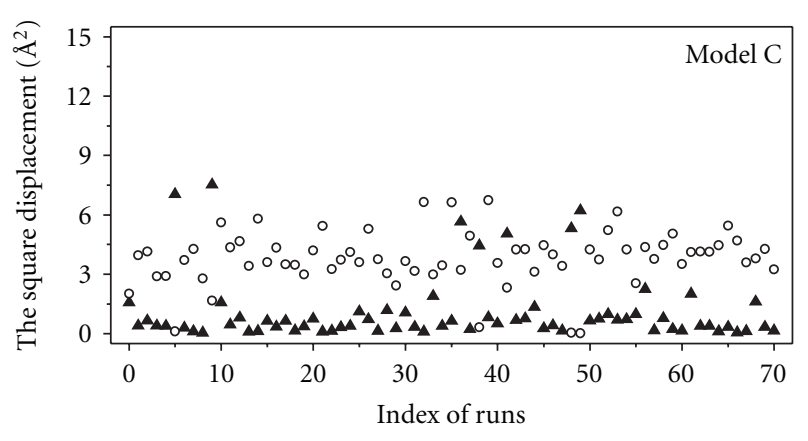

$\Delta \mathrm{B}$

$\circ \mathrm{C}$

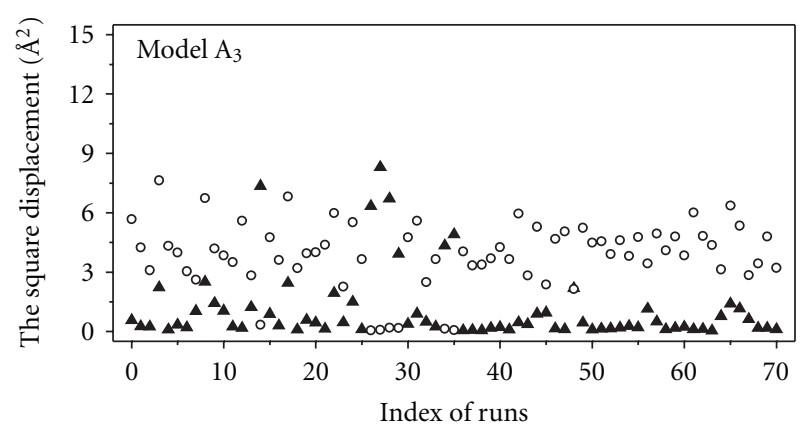

(b)

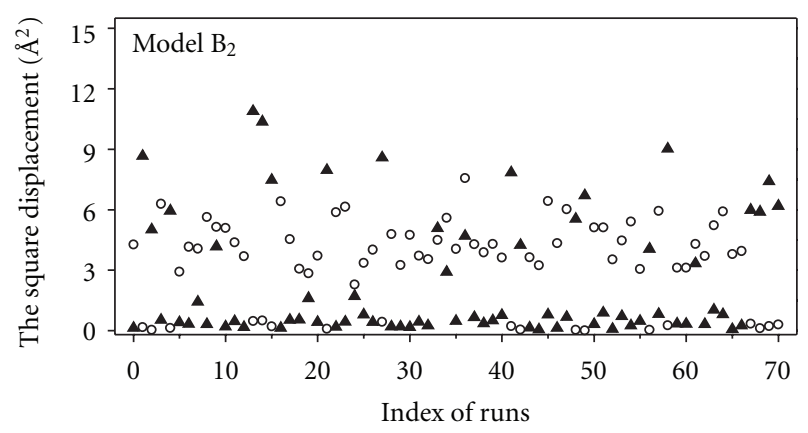

(d)

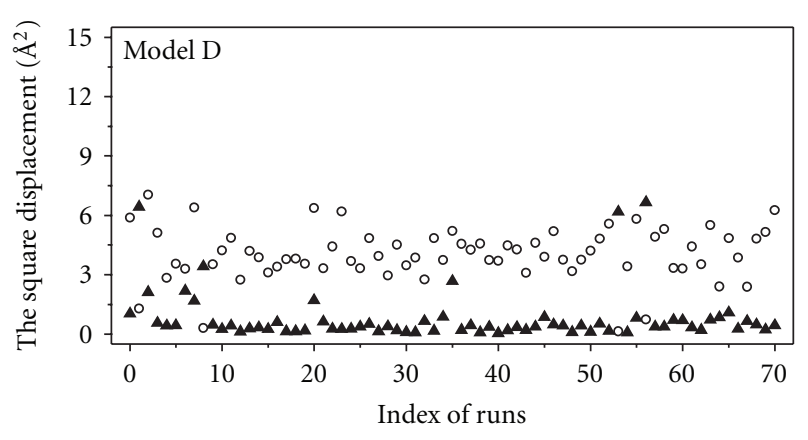

$\Delta \mathrm{B}$

$\circ \mathrm{C}$

(e)

(f)

Figure 9: The square displacements of $\mathrm{Co}$ and $\mathrm{B}$ atoms, $x_{\mathrm{Coi}}^{2}$ and $x_{\mathrm{Bi}}^{2}$ for the ith run of DA moving for $\mathrm{Co}_{100-x} \mathrm{~B}_{x}$.

\section{Conclusion}

A comprehensive study on local microstructure and tracer diffusion in amorphous alloys $\mathrm{Co}_{x} \mathrm{~B}_{100-x}$ and $\mathrm{Fe}_{80} \mathrm{P}_{20}$ has been carried out using SR technique and $n_{B}$-bubble statistic. Several conclusions can be made as given below.

(i) It was found that a large number of VBs function as diffusion vehicle. Once a neighboring atom jumps into $\mathrm{VB}$, the present $\mathrm{VB}$ disappears and it involves a collective movement of a large number of atoms. As a result, some bubbles located nearby transposes into $\mathrm{VB}$ and sometime the converse trend is observed when the VB transposes into small bubble. The number of VBs changes in the range of \pm 15 after atom jump and completing the relaxation. (ii) The number of VBs found in considered models depends essentially on the relaxation degree and density. From this follows that the decrease in diffusion constant for as-quenched sample in comparison with well-relaxed sample is ascribed to the decrease in VB's concentration in those samples. Regarding chemical composition the simulation reveals a largest number of VBs for the model $\mathrm{Co}_{70} \mathrm{~B}_{30}$. The model $\mathrm{Fe}_{80} \mathrm{P}_{20}$ contains a significantly larger number of VBs compared to model $\mathrm{Co}_{x} \mathrm{~B}_{100-x}$.

(iii) It was found two distinct diffusion mechanisms for as-quenched and well-relaxed samples of AMA. For first one the atom jump into $\mathrm{VB}$ and consequent relaxation leads to strong atomic arrangement which spreads over large region in the amorphous matrix. 
Such atomic movement has a collective character. In the case of well-relaxed sample, conversely the atomic arrangement upon DA jump locates in a small volume. Such diffusion mechanism likes the usual hoping mechanism via vacancies.

\section{Acknowledgment}

The authors are grateful for support by the NAFOSTED of Ministry of Science and Technology Vietnam.

\section{References}

[1] Y. Limoge, "Activation volume for diffusion in a metallic glass," Acta Metallurgica Et Materialia, vol. 38, no. 9, pp. 1733-1742, 1990.

[2] J. Horváth, J. Ott, K. Pfahler, and W. Ulfert, "Tracer diffusion in amorphous alloys," Materials Science and Engineering, vol. 97, pp. 409-413, 1988.

[3] J. Pavlovsky, W. Ulfert, and W. Frank, "Self-diffusion of Co in amorphous CoNbB during isothermal crystallization," Materials Chemistry and Physics, vol. 36, no. 3-4, pp. 383-388, 1994.

[4] W. Frank, J. Horváth, and H. Kronmüller, "Diffusion mechanisms in amorphous alloys," Materials Science and Engineering, vol. 97, pp. 415-418, 1988.

[5] Vo Van Hoang, "Computer simulation of the effects of B and $\mathrm{P}$ concentrations on microstructure in amorphous $\mathrm{Fe}-\mathrm{B}$ and Fe-P alloys," Physica B, vol. 348, no. 1-4, pp. 347-352, 2004.

[6] P. K. Hung, P. H. Kien, and L. T. Vinh, "Evidence of 'microscopic bubbles' and a new diffusion mechanism for amorphous alloys," Journal of Physics Condensed Matter, vol. 22, no. 3, Article ID 035401, 2010.

[7] S. Flege, U. Fecher, and H. Hahn, "Diffusion in amorphous NiZrAl alloys," Journal of Non-Crystalline Solids, vol. 270, no. 1-3, pp. 123-128, 2000.

[8] T. Schuler, J. Pavlovský, P. Scharwaechter, W. Ulfert, and W. Frank, "Change of the self-diffusion of Co in CoNbB during transition from the amorphous to the nanocrystalline phase," Nanostructured Materials, vol. 6, no. 5-8, pp. 863-867, 1995.

[9] A. Griesche, TH. Zumkley, M. P. Macht, S. Suzuki, and G. Frohberg, "Diffusion of PdCuNiP alloys from the amorphous to the liquid state," Materials Science and Engineering A, vol. 375-377, no. 1-2, pp. 285-287, 2004.

[10] S. Chakravarty, M. Gupta, A. Gupta et al., "Fe and N selfdiffusion in amorphous FeN: a SIMS and neutron reflectivity study," Acta Materialia, vol. 57, no. 4, pp. 1263-1271, 2009.

[11] A. van den Beukel and J. Sietsma, "Flow defects and diffusion defects in metallic glasses," Materials Science and Engineering A, vol. 134, pp. 935-938, 1991.

[12] A. K. Tyagi, M. P. Macht, and V. Naundorf, "Diffusion coefficients of $\mathrm{Ni}$ in FeNiB metallic glass," Acta Metallurgica Et Materialia, vol. 39, no. 4, pp. 609-617, 1991.

[13] W. Frank, A. Hörner, P. Scharwaechter, and H. Kronmüller, "Diffusion in amorphous metallic alloys," Materials Science and Engineering A, vol. 179-180, no. 1, pp. 36-40, 1994.

[14] R. S. Averback, "Defects and diffusion in amorphous alloys," MRS Bulletin, p. 47, November 1991.

[15] Y. Limoge, "Microscopic and macroscopic properties of diffusion in metallic glasses," Materials Science and Engineering A, vol. 226-228, pp. 228-236, 1997.

[16] P. K. Hung, H. V. Hue, and L. T. Vinh, "Simulation study of pores and pore clusters in amorphous alloys CoB and FeP,"
Journal of Non-Crystalline Solids, vol. 352, no. 30-31, pp. 33323338, 2006.

[17] Y. Limoge and G. Brebec, "Relation between viscosity and diffusion in amorphous metallic alloys," Acta Metallurgica, vol. 36, no. 3, pp. 665-673, 1988.

[18] A. Zhu, G. J. Shiflet, and S. J. Poon, "Diffusion in metallic glasses: Analysis from the atomic bond defect perspective," Acta Materialia, vol. 56, no. 14, pp. 3550-3557, 2008.

[19] L. D. Van Ee, B. J. Thijsse, and J. Sietsma, "Evidence for twolevel states and cooperative atomic jumps in a computer model of amorphous $\mathrm{Ni}_{81} \mathrm{~B}_{19}$," Materials Science and Engineering $A$, vol. 226-228, pp. 296-300, 1997.

[20] V. Naundorf, M. P. Macht, A. S. Bakai, and N. Lazarev, "The pre-factor, D, of the diffusion coefficient in amorphous alloys and in grain boundaries," Journal of Non-Crystalline Solids, vol. 224, no. 2, pp. 122-134, 1998.

[21] H. Kronmüller, W. Frank, and A. Hörner, "Diffusion and structural-relaxation mechanisms in metallic glasses," Materials Science and Engineering A, vol. 133, pp. 410-414, 1991.

[22] D. K. Belashchenko, V. V. Hoang, and P. K. Hung, "Computer simulation of local structure and magnetic properties of amorphous Co-B alloys," Journal of Non-Crystalline Solids, vol. 276, no. 1, pp. 169-180, 2000.

[23] P. Lamparter et al., "X-ray and neutron diffraction studies on amorphous transition metal-boron alloys (Fe-B, Co-B, Ni-B)," Zeitschrift für Naturforschung, vol. 6, p. 165, 1981.

[24] G. S. Chadha, M. Sakata, N. Cowlam, and H. A. Davies, "A diffraction study of $\mathrm{Co}_{81.5} \mathrm{~B}_{18.5}$ binary metallic glass," Physica Status Solidi (A), vol. 63, no. 2, pp. 625-630, 1981.

[25] Y. Waseda and H. S. Chen, "A structural study of metallic glasses containing boron (Fe-B, Co-B and Ni-B)," Physica Status Solidi A, vol. 49, no. 1, pp. 387-392, 1978.

[26] Y. Wasede, The Structure of Non-Crystalline Materials, McGraw-Hill, New York, NY, USA, 1980.

[27] Y. Waseda, "The structure of liquids, amorphous solids and solid fast ion conductors," Progress in Materials Science, vol. 26, no. 1, pp. 1-122, 1981.

[28] T. Fujiwara and Y. Ishii, "Structural analysis of models for the amorphous metallic alloy Fe P," Journal of Physics F, vol. 10, no. 9, article no. 008, pp. 1901-1911, 1980.

[29] J. Pavlovsky, W. Ulfert, and W. Frank, "Self-diffusion of Co in amorphous CoNbB during isothermal crystallization," Materials Chemistry and Physics, vol. 36, no. 3-4, pp. 383-388, 1994.

[30] W. Dörner and H. Mehrer, "Tracer diffusion and thermal stability in amorphous Co-Zr and their relevance for solidstate amorphization," Physical Review B, vol. 44, no. 1, pp. 101-114, 1991.

[31] J. Horvath and H. Mehrer, "Tracer diffusion of ${ }^{59} \mathrm{Fe}$ in amorphous $\mathrm{Fe}_{40} \mathrm{Ni}_{40} \mathrm{~B}_{20}$," Crystal Lattice Defects and Amorphous Materials, vol. 13, p. 1, 1986.

[32] R. W. Cahn, J. E. Evetts, J. Patterson, R. E. Somekh, and C. K. Jackson, "Direct measurement by secondary-ion mass spectrometry of self-diffusion of boron in FeNiB glass," Journal of Materials Science, vol. 15, no. 3, pp. 702-710, 1980.

[33] J. Horvath, K. Freitag, and H. Mehrer, "Tracer diffusion of implanted ${ }^{32} \mathrm{P}$ in amorphous $\mathrm{Fe}_{40} \mathrm{Ni}_{40} \mathrm{~B}_{20}$," Crystal Lattice Defects and Amorphous Materials, vol. 13, p. 15, 1986.

[34] D. R. Baer, L. R. Pederson, and M. T. Thomas, "Phosphorus diffusion in FeNi-based amorphous alloys," Materials Science and Engineering, vol. 48, no. 2, pp. 283-290, 1981. 

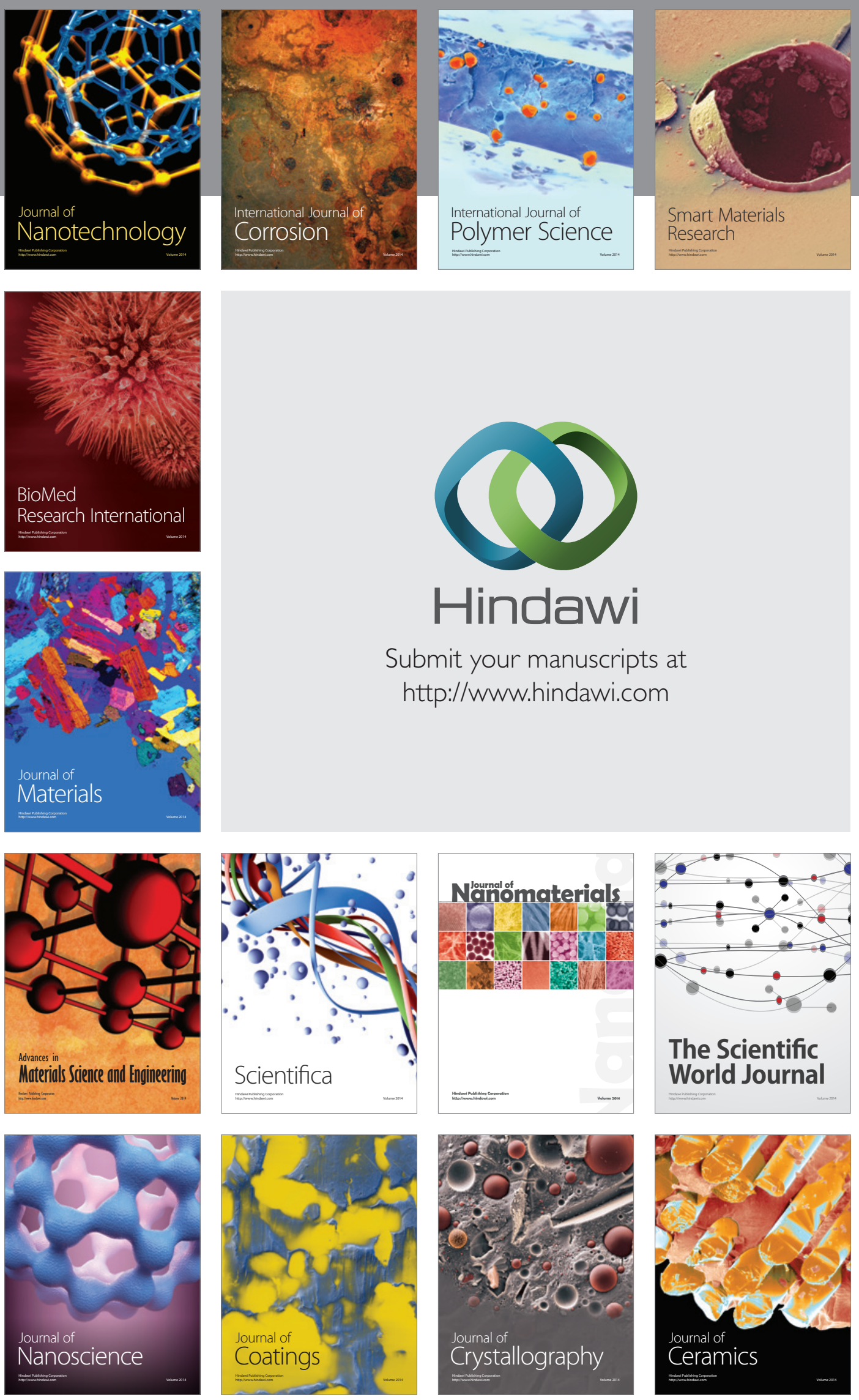

The Scientific World Journal

Submit your manuscripts at

http://www.hindawi.com

\section{World Journal}

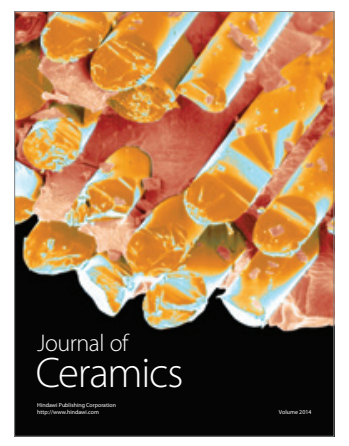

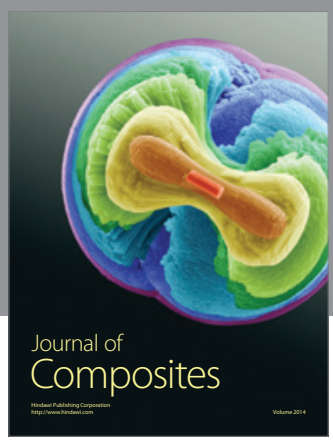
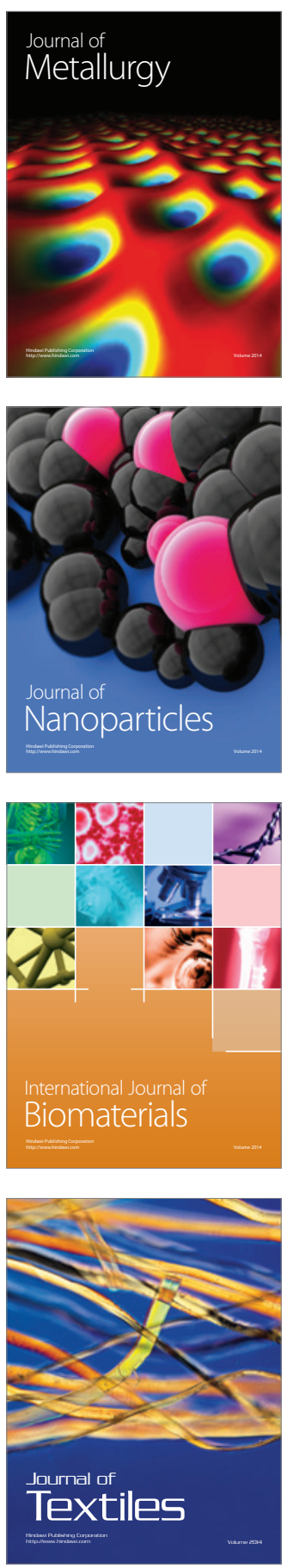